\title{
A Direct Non-destructive Method for Determination of Sulfur in Ore Samples Using EDXRF Spectrometry
}

\author{
Buddhadev Kanrar, *** S. Sanjay Kumar, * S. Mondal, *** N. L. Mishra,* and Sangita Dhara*,**i \\ *Fuel Chemistry Division, Bhabha Atomic Research Centre, Mumbai, 400085, India \\ **Homi Bhabha National Institute, Anushakti Nagar, Mumbai, 400094, India \\ ***Materials Processing and Corrosion Engineering Division, Bhabha Atomic Research Centre, Mumbai, \\ 400085, India
}

\begin{abstract}
A method has been developed for direct non-destructive energy dispersive X-ray fluorescence (EDXRF) determination of sulfur in solid uranium ores and intermediates, obtained from the alkaline recovery process for uranium from its ores. The method involves thorough grinding of a few $\mathrm{mg}$ of solid powder samples to fine particle size and mixing the fine powder thus obtained with a few drops of $10 \%$ collodion solution in amyl acetate to make a paste. A very small amount of this paste was transferred with the help of the pestle tip, spread uniformly in the form of thin slurry on Mylar films, and dried to make very thin sample specimens on thin Mylar film supports. These specimens were presented for EDXRF measurements. A calibration plot was made by plotting the intensity ratios of $S K \alpha$ and Rayliegh scattered peak of the excitation source $(\mathrm{Ge} K \alpha)$ against sulphur percent in the certified reference materials (CRMs). It was found that the precision obtained using this methodology was within $5 \%( \pm 1 \sigma)$ and the deviation of the EDXRF analytical results from the expected values of CRM was within 7\%. The developed method was successfully applied for the determination of sulfur in the samples obtained from the different stages of the uranium ore processing using alkaline based leaching method.
\end{abstract}

Keywords EDXRF, sulfur, uranium ores, Rayleigh scattering

(Received November 9, 2020; Accepted December 24, 2020; Advance Publication Released Online by J-STAGE January 1, 2021)

\section{Introduction}

The most important application of uranium is its use as nuclear fuel in the reactors for the generation of electricity. Worldwide, the $\mathrm{U}$ content in most of the uranium ores varies from 0.02 to $0.2 \%$ as $\mathrm{U}_{3} \mathrm{O}_{8}$. Recovery of $\mathrm{U}$ from the ores is a very important step in the nuclear fuel cycle, which involves dissolution of the ores and extraction of uranium from the ores. In view of the

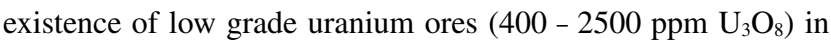
India, the recovery process of uranium from its ores need to be as efficient as possible. ${ }^{1}$ Uranium is extracted from ores either by acidic leaching in sulfuric acid or by alkaline leaching in sodium carbonate. ${ }^{1,2}$ In India, carbonate based uranium ores are present in Tummalapalle in Andhra Pradesh and Gogi in Karnataka, which require the alkaline leaching process. Under ambient temperature and atmospheric pressure, the gangue minerals present in the ores play a very insignificant role. However, under elevated temperature and pressure conditions, some of these minerals are susceptible to dissolution. Pyrite $\left(\mathrm{FeS}_{2}\right)$ is one of the most common sulphide ores, usually present up to $2 \%$ along with uranium ores. During the alkaline leaching process, $\mathrm{FeS}_{2}$ forms $\mathrm{Fe}(\mathrm{OH})_{2}$, gets dissolved and starts leaching with the carbonate along with uranium. The dissolution of

$\dagger$ To whom correspondence should be addressed.

E-mail: sdhara@barc.gov.in

N. L. M., Retired Scientific Officer. uranium ores associated with pyrites require elevated temperatures $\left(<100^{\circ} \mathrm{C}\right)$ in oxidizing conditions for complete dissolution. The presence of a significant amount of sulfide in the ores results in excessive consumption of the leachants and the reactive conditions required for its dissolution, in turn, dissolve some other gangue materials also in the alkaline leachants. This results in increased total dissolved solute (TDS) content in the leach liquor. These undesired materials in the leach liquor hinder the effective recovery of uranium from the ores $^{1}$. In view of the above discussions regarding the importance of sulfur in the alkaline processing of uranium ores for uranium recovery, it is essential to determine the sulfur content in the ores as well as in the intermediate residues obtained at different stages of the leaching process for optimizing the leaching conditions. Such determinations shall be beneficial for adopting an efficient method of uranium recovery by the alkaline dissolution process. This requires a simple, fast and preferably a non destructive method of sulfur determination in uranium ores and processing materials on a routine basis.

The conventional methods used for the determination of sulfur include ion chromatography, ${ }^{3}$ and for analysis in solid samples directly, combustion of the samples in air atmosphere to form $\mathrm{SO}_{2}$, followed by infrared detection of $\mathrm{SO}_{2}$ for quantification is used. ${ }^{4}$ However these techniques require either dissolution of the samples or heating the samples to high temperatures, which make these methods time consuming as well as tedious. Sometimes, samples are pyrohydrolyzed and the sulfur content is collected in a condensate and then analyzed. Pyrohydrolysis 
of solid samples for separation of the matrix, followed by the determination of sulfur by inductively coupled plasma optical emission spectrometry (ICP OES) and ion chromatography (IC) are also used. ${ }^{5}$ Recently, a triple quadrupole inductively coupled plasma mass spectrometry method was reported for the determination of sulfur and phosphorus in UOC samples. Here also the samples are dissolved in $\mathrm{HNO}_{3}$ using normal as well as microwave digestion methods. ${ }^{6}$ However, for sulfur determinations in uranium ores on a routine basis, a simple and non destructive method will be more suitable.

$\mathrm{X}$-ray fluorescence (XRF) is a fast, versatile and efficient multi-elemental determination method suitable for solid samples, with no requirement of sample dissolution. ${ }^{7}$ Because of its numerous advantages, it is being widely used in several areas for research and routine sample analysis. ${ }^{8-10}$ However, XRF suffers from severe matrix effect and requires matrix matched standards for quantification. There are many methods for minimizing the matrix effect in XRF analysis. ${ }^{8}$ Presentation of the sample in the form of thin specimens is one of the methods to minimize the matrix effect to some extent. ${ }^{11}$ Further, in XRF, the determination of low atomic number $(\mathrm{Z})$ elements in the presence of medium and high $\mathrm{Z}$ matrix elements is another challenge. ${ }^{12}$. The fluorescence yields of the characteristic $\mathrm{X}$-ray analytical lines of low $\mathrm{Z}$ elements are considerably less (e.g. 0.06 for sulfur). In addition, the low energy $\mathrm{X}$-rays emitted from low $\mathrm{Z}$ elements are absorbed appreciably by the medium and high $\mathrm{Z}$ elements present in the sample, which have high mass absorption cross section. These factors lead to a further decrease in the fluorescent intensity of the low $\mathrm{Z}$ elements when analyzed by XRF. Moreover, the $\mathrm{X}$ ray lines of medium and high $\mathrm{Z}$ elements interfere severely with the $\mathrm{K}$ lines of low $\mathrm{Z}$ elements. The Mo L line $(2.29 \mathrm{keV})$ and $\mathrm{Pb} \mathrm{M}$ line $(2.34 \mathrm{keV})$ interferes with $\mathrm{S} K \alpha(2.31 \mathrm{keV})$. The presence of these elements complicates the quantification process. So for XRF determination of sulfur having characteristic X-ray energy of $2.31 \mathrm{keV}(\mathrm{S} K \alpha)$, the above stated problems need to be addressed.

In $\mathrm{U}$ ore samples, the quantity of sulfur is generally less than $2 \%$, hence a proper sample preparation procedure, which will take care of the low sulfur concentration as well as the matrix effect, is needed. Dissolving the sample has many disadvantages such as addition of corrosive acids, incorporation of impurities and loss of volatile elements. Moreover, it is a time consuming and tedious process. So, non-destructive methods are preferred to aviod the above stated problems. Presentation of the sample as a thin paste and quantification using the primary scattered peaks of the target element is a very simple and well known method for non destructive analysis of solid samples without addition of any internal standard. ${ }^{13}$ This method of quantification is based on the fact that the intensities of the characteristic $\mathrm{X}$-rays of the analyte element and that of the scattered peak vary in a similar way as the composition of the matrix. In fact, it is reported that for the elimination of the matrix effect, both Compton and Rayleigh scattered peaks can be used. ${ }^{13,14}$ Since the ratio of the characteristic X-ray peak and the scattered peak is less sensitive towards variation in composition and amount of the sample, it can be used for the correction of the matrix effects. ${ }^{13}$

This paper describes the development of a direct nondestructive energy dispersive (ED) XRF method for determination of sulfur in samples obtained during various stages of the alkaline leaching process of uranium ores. The method is very simple and is suitable for routine analysis of ore samples.
Table 1 Details of the ore standards used for calibration

\begin{tabular}{clc}
\hline S. No & Standard & Certified values of $\mathrm{S} \pm 1 \sigma, \%$ \\
\hline 1 & GS305-10 & $0.51 \pm 0.04$ \\
2 & GS307-3 & $1.03 \pm 0.09$ \\
3 & GS302-5 & $1.98 \pm 0.10$ \\
\hline
\end{tabular}

The values in \pm are standard deviation values $(1 \sigma)$.

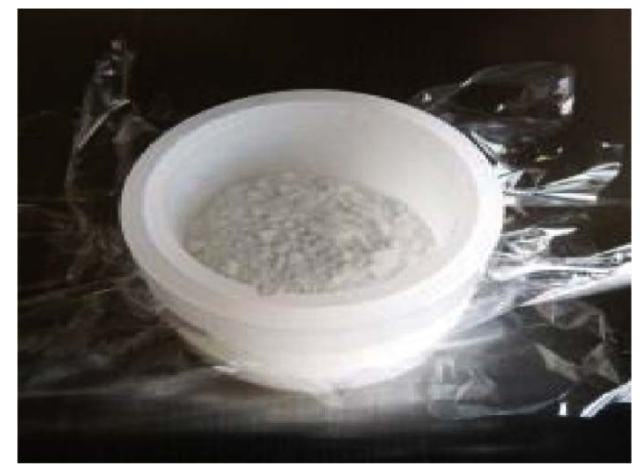

Fig. 1 The EDXRF sample cup with thin Mylar film stretched by the PVC rings.

\section{Experimental}

\section{Sample preparation}

The calibration plots for EDXRF analysis were made using CRMs (Certified Reference Materials) for sulfur from "GEOSTATS PTY LTD", Australia. The sulfur content in these ore standards were up to $2 \%$. The details of these standards are given in Table 1. About $100 \mathrm{mg}$ of the solid ore CRMs were first finely ground in a clean pestle mortar for about $10 \mathrm{~min}$. A few drops of $10 \%$ collodion solution in amyl acetate were added to this fine powder in the mortar itself. Collodion is a very sticky solvent, which sticks to any surface quite strongly after drying. The collodion mixed standard powders in the mortar were ground again for about $5 \mathrm{~min}$. Uniform slurries of the standards were thus obtained. Each standard was processed in separate mortars to avoid cross contamination. The well mixed slurries were again ground with the tip of the pestle and the pestle tip was just touched at the center of $4 \mu \mathrm{m}$ thin Mylar films stretched uniformly over PET sample cups, used for EDXRF measurement of liquid samples. The Mylar films were uniformly stretched over these cups by enclosing them on the cups and pressed by the same size PVC rings as shown in Fig. 1. The pestle was touched and slightly rubbed on the Mylar film from the inside of the cup so that a few mg of the sample were transfered on the Mylar film. The specimens formed were left to dry in ambient atmosphere. After drying, a thin uniform film of the standard was formed on the Mylar. For each standard and sample, three such specimens were made and presented for XRF measurements. The samples obtained from the different stages of the uranium ore processing, using alkaline based leaching method, were also processed similarly and analyzed after measuring their EDXRF spectra in the similar manner.

\section{Instrumentation}

For EDXRF measurements, a Jordan Valley EX-3600TEC EDXRF spectrometer having Rh X-ray tube target was used. The instrument is equipped with a twelve-position sample 
chamber and can measure the samples sequentially. There is a provision for using different X-ray filters, placed between the $\mathrm{X}$-ray tube and sample holder. The filters can be used for reducing the background in a particular energy range depending on the elements of interest. Three secondary targets, Gd, Ge and $\mathrm{Al}$, are provided to excite elemental X-ray lines efficiently and reduce the spectral background. Maximum power capacity of the EDXRF spectrometer is $50 \mathrm{~W}$ with maximum voltage and current of $50 \mathrm{kV}$ and $3000 \mu \mathrm{A}$, respectively. In order to excite $\mathrm{S} K \alpha$ efficiently and reduce the spectral background, $\mathrm{Ge}$ secondary target was used for excitation of the samples by $\mathrm{Ge}$ $K \alpha(9.89 \mathrm{keV})$ generated from the secondary target. The current and voltage applied to the X-ray tube were $2000 \mu \mathrm{A}$ and $20 \mathrm{kV}$, repectively. The $\mathrm{X}$-rays were detected by a peltier cooled Si-PIN diode detector, having a resolution of $140 \mathrm{eV}$ (FWHM) at $5.9 \mathrm{keV}$. All measurements were carried out in air atmosphere. The measurement time was $1000 \mathrm{~s}$ for each specimen.

All the EDXRF spectra were processed using the EDXRF 32 program from Ital-Structures, Italy. It is an X-ray peak profile fitting program that is based on nonlinear least-squares fitting using Marquardt algorithm. ${ }^{15}$ This program was used for processing and calculating the intensities of $\mathrm{S} K \alpha$ and Ge $K \alpha$ lines. In this study we observed that the profile fitting of $\mathrm{Ge} K \alpha$ Rayleigh scattered peak was more precise compared to that of Compton scattered peak using this program. Hence, we have taken the Rayleigh scattered peak for normalizing the analyte peak intensity.

\section{Results and Discussion}

Though EDXRF is a well established method for non-destructive analysis of a variety of samples, determination of sulfur in uranium ore, having medium and high $\mathrm{Z}$ matrix elements is quite challenging due to several reasons discussed above. Presentation of solid samples in the form of pellets requires a larger sample amount. As the pellets are of infinite thickness, during XRF analysis, high background and severe matrix effects are encountered. In order to overcome these problems, an EDXRF method with thin specimens was developed in our laboratory. ${ }^{11}$ The thin film specimens were made by drying the solution samples on the scotch tape. As a thin film of the solid sample on the thin support was made by placing the slurry of the sample on Mylar, the matrix effects were minimized but not completely removed. The remaining matrix effect can be taken care by using the intensity ratio of the analyte peak and Compton or Rayleigh scattered peak of the target. Hence the calibration plot was made using the intensity ratios of analyte peak and the scattered Rayleigh peak of the exciting radiation $(\mathrm{Ge} K \alpha)$. The use of such ratio for quantification shall mitigate the matrix effect to a large extent as this ratio is insensitive to the variation in the matrix composition, but depends on the concentration of the trace analytes. In addition, such ratio shall also mitigate the error due to sample geometry and area of the sample exposed to the exciting $\mathrm{X}$ ray beam. Also, as stated above for efficient excitation of $\mathrm{S} K \alpha$ and reducing the background further, $\mathrm{Ge}$ secondary target exciation shall be beneficial. All the above features were included in the present work for development of an accurate, fast and simple method for the determination of sulfur in ore matrices. The EDXRF spectrum of a CRM containing $2 \%$ sulfur excited by Ge secondary target is shown in Fig. 2. The X-ray peaks of $\mathrm{Si}, \mathrm{Ar}, \mathrm{K}, \mathrm{Ti}, \mathrm{V}, \mathrm{Cr}, \mathrm{Fe}, \mathrm{Cu}$ and $\mathrm{Zn}$ along with the sulfur peak were observed in the EDXRF spectrum of the standard. The U M lines (3.17 keV) have interference with $\mathrm{K} K \alpha$ lines $(3.31 \mathrm{keV})$ and are shown in the

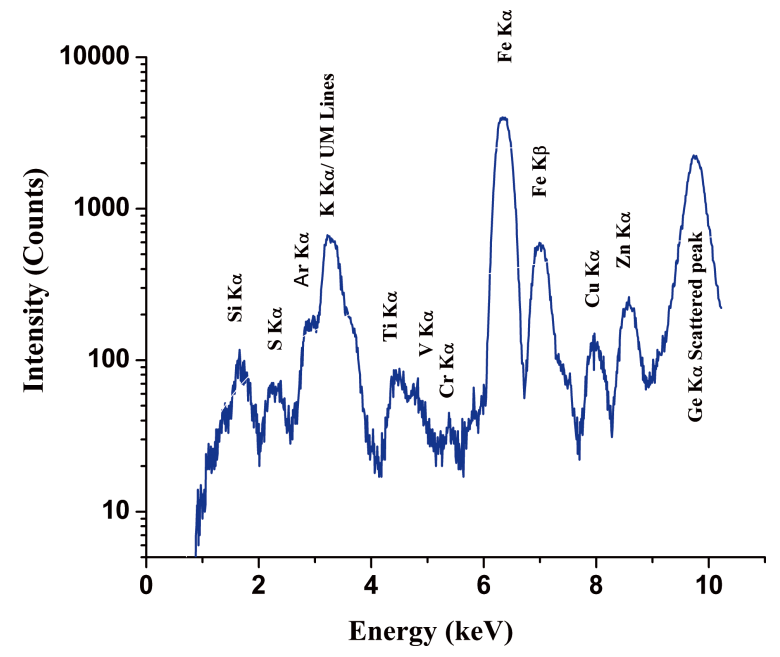

Fig. 2 EDXRF spectrum of an ore CRM containing $2 \%$ sulfur excited using Ge secondary target operated at $2000 \mu \mathrm{A}$ and $20 \mathrm{kV}$.

figure. The EDXRF detection limit of sulfur was determined using the standard containing $0.51 \pm 0.04 \%$ sulfur and measurement time of $1000 \mathrm{~s}$ and it was found to be $0.03 \%$.

The samples obtained from the different stages of the uranium ore processing contained traces of $\mathrm{U}$ and $\mathrm{Pb}$. Using $\mathrm{Ge}$ secondary target, the $\mathrm{M}$ lines of $\mathrm{U}$ and $\mathrm{Pb}$ can be excited. The $\mathrm{Pb} \mathrm{M}$ lines $(2.35 \mathrm{keV})$ interfere with $\mathrm{S} \mathrm{K}$ line $(2.31 \mathrm{keV})$. However, as $\mathrm{Pb}$ is present in trace amounts of a few ppm and the $\mathrm{M}$ lines of $\mathrm{Pb}$ have very low fluorescence yield (0.03), the intensity of $\mathrm{Pb} \mathrm{M}$ lines will be very low. ${ }^{16}$ Moreover, the EDXRF 32 program was used for the elemental X-ray peak fitting and this program can take care of any X-ray interferences by profile fitting and gives individual intensities of interfereing elemental $\mathrm{X}$ ray peaks. ${ }^{15}$ So even if some traces of $\mathrm{Pb}$ were present in the sample, the EDXRF 32 program can calculate the area of $\mathrm{S} \mathrm{K}$ peak and $\mathrm{Pb} \mathrm{M}$ line contributions, separately.

For making the calibration plot, the intensity ratios of $\mathrm{S} K \alpha$ and scattered peak of Ge $K \alpha$ was plotted against the sulfur percentage in the CRMs. The calibration was found to be linear and is shown in Fig. 3. The equation obtained was

$$
y=136.26 x+0.033
$$

and the $R^{2}=0.9958$. Despite variation in the amount of sample that was deposited on the Mylar film support, a linear calibration was obtained indicating that the ratio of characteristic X-ray line of the analyte and scattered peak is not affected by any other parameter except the sulfur concentration. So the scattered peak acts as an external standard and takes care of the matrix effect occuring in the sample. In order to validate the developed method, another set of the standards specimens was prepared and analyzed using the above equation. The analytical results along with the accuracy and precision obtained are tabulated in Table 2. The precision obtained was within $5 \%(\mathrm{RSD}, \pm 1 \sigma)$ and the deviation of the EDXRF analytical results from the expected values was within $7 \%$. Using the above calibration plot, the sulfur contents in some of the ore samples obtained during uranium ore processing were determined. The samples were taken from different stages of the ore processing process and analyzed in a similar manner as the standards. Different types of sample, such as finely ground ore samples without any processing, residues of samples before attaining the final 
temperature and pressure, and sample residues obtained after $6 \mathrm{~h}$ of leaching at the temperature of $130^{\circ} \mathrm{C}$ with $4 \mathrm{~kg} / \mathrm{cm}^{2} \mathrm{O}_{2}$ pressure, were analyzed using the developed procedure. The analytical results obtained are given in Table 3 .

Thus, a simple, non-destructive, direct EDXRF method for sulfur determination in uranium ore samples without the requirement of any laborious sample preparation procedure, such as dissolution, separation or pressed pelletization, was developed. The developed methodology will be useful for sulfur determination in not only uranium ores, but also in other similar matrices requiring sulphur determinations in a fast and simple manner e.g. coal, soil, etc. The sample remains in the same form as taken because the collodion evaporates after drying without reacting with the sample.

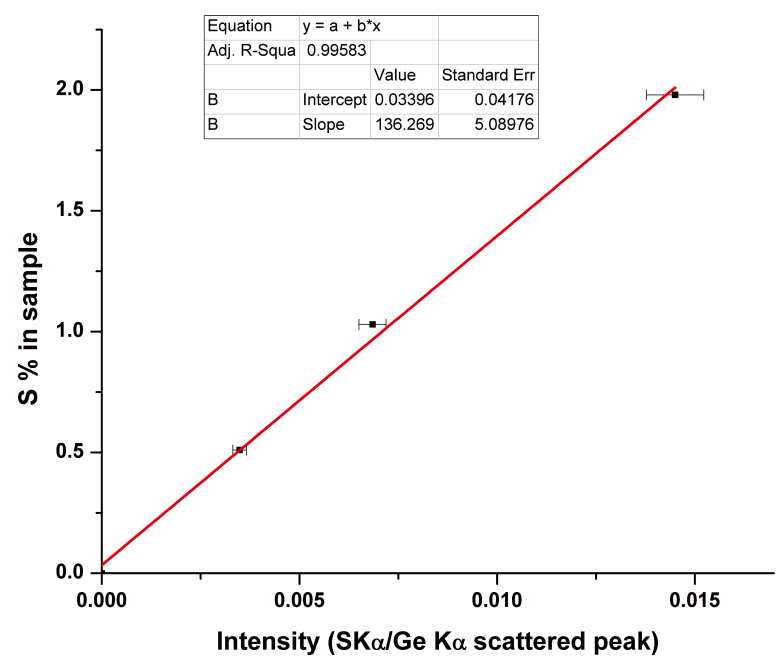

Fig. 3 Calibration plot for EDXRF determination of sulfur in ores samples.

Table 2 Comparison of the analytical results obtained using EDXRF determined and certified values

\begin{tabular}{cccc}
\hline S. No & $\begin{array}{c}\text { Certified values of } \\
\mathrm{S} \pm 1 \sigma(\text { in } \%) \\
\mathbf{A}\end{array}$ & $\begin{array}{c}\text { EDXRF determined } \\
\mathrm{S} \pm 1 \sigma(\text { in } \%) \\
\mathbf{B}\end{array}$ & $\begin{array}{c}\text { EDXRF }(\mathbf{B}) / \\
\text { certified (A) }\end{array}$ \\
\hline 1 & $1.03 \pm 0.09$ & $1.10 \pm 0.05$ & 1.07 \\
2 & $1.98 \pm 0.10$ & $1.90 \pm 0.1$ & 0.96 \\
\hline
\end{tabular}

The values in \pm are standard deviation values $(1 \sigma)$.

\section{Conclusions}

A simple non-destructive direct EDXRF method for sulfur determination in solid ore samples was developed without addition of any internal standard. The method utilizes the calibration plot made by plotting intensity ratio of $\mathrm{S} K \alpha$ and Rayleigh scattered peak of the exciting radiation $(\mathrm{Ge} K \alpha)$ against the sulfur percentage present in the standards. The EDXRF specimens were presented in the form of thin film made by mixing the ore powders in collodion solution. Use of thin film reduced the background considerably and took care of the matrix effect, whereas use of secondary target as excitation source made the excitation efficient with decrease in the background. The developed method gave satisfactory results for the analysis of sulfur in ore standards containing sulfur within $2 \%$. The precision obtained was within $5 \%(\mathrm{RSD}, \pm 1 \sigma)$ and the deviation of the analytical results from the expected values was within $7 \%$. The developed method was sucessfully applied for the determination of sulfur in the different stages of the uranium ore processing using an alkaline-based leaching method.

\section{Acknowledgements}

The authors are thankful to Dr. P. K. Pujari, Director, Radiochemistry and Isotope Group and Dr. S. Kannan, Head of the Fuel Chemistry Division, for their interest in the present work.

\section{References}

1. T. Sreenivas and J. K. Chakravartty, Trans. Indian Inst. Met., 2016, 69, 3.

2. K. Anand Rao, B. Paul, and T. Sreenivas, Trans. Indian Inst. Met., 2020, 73, 2069.

3. C. D. Chriswell, D. R. Mroch, and R. Markuszewski, Anal. Chem., 1986, 58, 319.

4. S. Terashima, Anal. Chim. Acta, 1978, 101, 25.

5. T. S. Nunes, C. C. Muller, P. Balestrin, A. L. H. Muller, M. F. M. P. de Azevedo Mello, and E. I. Muller, Anal. Methods, 2015, 7, 2129.

6. N. D. Fletcher, B. T. Manard, D. A. Bostick, W. D. Bostick S. C. Metzger, B. W. Ticknor, K. T. Rogers, and C. R. Hexel, Talanta, 2021, 221, 121573.

7. R. E. Van Griken and A. Markowicz, "Handbook of X-ray Spectrometry", 1993, Marcel and Dekker Inc.

8. E. Marguí, I. Queralt, and M. Hidalgo, Trends Anal. Chem., 2009, 28, 362 .

9. V. Singh, J. Appl. Spectrosc., 2017, 84, 289.

Table 3 EDXRF determined concentration of sulfur in some residues of uranium ore obtained at different processing stages

\begin{tabular}{llrrr}
\hline \multirow{2}{*}{ Sample } & \multicolumn{1}{c}{ Leaching stage and sample description } & $\begin{array}{c}\text { EDXRF determined } \\
\text { sulfur, } \%\end{array}$ & $\begin{array}{c}\text { Standard deviation, } \\
1 \sigma\end{array}$ & $\begin{array}{c}\text { Relative standard } \\
\text { deviation, } \%\end{array}$ \\
\hline Feed & Ground ore & 0.64 & 0.05 & 7.8 \\
1T1 & Residue samples before reaching the required temperature and pressure & 0.70 & 0.10 & 14.3 \\
2T1 & Residue samples before reaching the requiredtemperature and pressure & 0.70 & 0.10 & 14.3 \\
1T9 & After 6 $\mathrm{h}$ of leaching, $4 \mathrm{~kg} / \mathrm{cm}^{2} \mathrm{O}_{2}$ pressure, $130^{\circ} \mathrm{C}$ & 0.72 & 0.05 & 6.9 \\
2T9 & After $6 \mathrm{~h}$ of leaching, $6 \mathrm{~kg} / \mathrm{cm}^{2} \mathrm{O}_{2}$ pressure, $130^{\circ} \mathrm{C}$ & 0.47 & 0.07 & 14.9 \\
3T9 & After $6 \mathrm{~h}$ of leaching, $10 \mathrm{~kg} / \mathrm{cm}^{2} \mathrm{O}_{2}$ pressure, $130^{\circ} \mathrm{C}$ & 0.50 & 0.10 & 20 \\
\hline
\end{tabular}

a. Residue obtained after leaching of uranium from uranium ore samples at different stages of leaching. 
10. J. Laursen, B. T. Vestergaard, N. Pind, K. Karlsen, and H. 14. Z. H. Kalman, Anal. Chem., 1962, 34, 946. C. B. Hansen, X-Ray Spectrom., 2001, 30, 186.

11. B. Kanrar, K. Sanyal, N. L. Misra, and S. K. Aggarwal, Spectrochim. Acta, Part B, 2014, 101, 130.

12. S. Dhara, N. L. Misra, and S. K. Aggarwal, X-Ray Spectrom., 2014, 43, 108.

13. A Marowicz, Pramana, 2011, 76, 321.

15. S. Dhara, A. Khooha, A. Kumar Singh, M. K. Tiwari, and N. L. Misra, Spectrochim. Acta, Part B, 2018, 144, 87.

16. J. H. Hubbell, P. N. Trehan, N. Singh, B. Chand, D. Mehta, M. L. Garg, R. R. Garg, S. Singh, and S. Puri, J. Phys. Chem. Ref. Data, 1994, 23, 339. 